\title{
SELF-HOMOTOPY EQUIVALENCES OF THE TOTAL SPACES OF A SPHERE BUNDLE OVER A SPHERE
}

\author{
Dedicated to Professor Minoru Nakaoka on his 60th birthday
}

\author{
By Seiya Sasao
}

\section{$\S 1$. Introduction.}

In this paper we study the group of homotopy classes of self-homotopy equivalences, $\mathcal{E}(X)$, for the total space of a $S^{m}$-bundle over $S^{n}$ with the condition:

$$
3<m+1<n<2 m-2 .
$$

J. W. Rutter determined this group for the case of $m=3$ and $n=7$ in [3], and also some generalizations of Rutter's result are given in [4] and [6]. Moreover Y. Nomura computed $\mathcal{E}(X)$ for real and complex Stiefel manifolds in [5]. Then our purpose is to obtain a generalization of these results in a some sense. Let $H$ be the natural representation:

$$
H: \mathcal{E}(X) \longrightarrow \text { Aut } H_{*}(X)
$$

which is defined by $H(f)=f_{*}$ and we denote by $\varepsilon_{+}(X)$ the kernel of $H$. Then we have an exact sequence

$$
\{1\} \longrightarrow \mathcal{E}_{+}(X) \longrightarrow \mathcal{E}(X) \underset{H}{\longrightarrow} \text { Aut } H_{*}(X) .
$$

Hence it is almost sufficient for us to determine $\varepsilon_{+}(X)$ and $H$-image.

Let $q: X \rightarrow S^{n}$ be the $S^{m}$-bundle with the characteristic class $\xi\left(\in \pi_{n-1}(S O(m+1))\right)$. James-Whitehead showed in [2] that $X$ has a $C W$-decomposition:

$$
X=S^{m} \bigcup_{\beta} e^{n} \bigcup_{\alpha} e^{m+n},
$$

where $\beta=p_{*}(\xi)$ for the usual projection $p: S O(m+1) \rightarrow S^{m}$.

Let $P_{n}^{m}(\beta)$ be the subgroup of $\pi_{n}\left(S^{m}\right)$,

$$
\left\{x \mid\left[\iota_{n}, x\right] \in \beta \circ \pi_{m+n-1}\left(S^{n-1}\right)\right\},
$$

and we denote by $\eta$ the generator of $\pi_{N+1}\left(S^{N}\right)$. We will prove

Received January 30, 1984 
Theorem 1. Suppose that $\left[\iota_{m+1}, E \beta\right] \circ \eta \equiv 0 \bmod E \beta \circ \pi_{m+n+1}\left(S^{n}\right)$. Then there exists an exact sequence

$$
\{0\} \longrightarrow H_{\xi} \longrightarrow \mathcal{E}_{+}(X) \longrightarrow G_{\xi} \longrightarrow\{0\},
$$

where

and

$$
H_{\xi}=\pi_{m+n}(X) /\left[\iota_{m}, \pi_{n+1}(X)\right] \cup\left\{\pi_{m+1}(X) \circ J(\xi)\right\}
$$

$$
G_{\xi}=P_{n}^{m}(\beta)\left[\{\beta \circ \eta\} \subset \pi_{n}\left(S^{m}\right) /\left\{\beta \circ \gamma_{i}\right\}\right. \text {. }
$$

Remark. For example, the assumption is always satisfied if $m=2 \bmod 4$ and $m \geqq 9$.

Theorem 2. Suppose $2 \beta=0$.

$$
\begin{aligned}
& H \text { - } m \text { mage }=Z_{2} \times Z_{\text {. }} \text { if } 2 J(\xi) \equiv 0 \text { and }\left[\iota_{m+1}, E \beta\right] \equiv 0 \bmod E \beta \circ \pi_{m+n}\left(S^{n}\right) \\
& H \text {-ımage }=Z_{2} \text { if elther }\left[\iota_{m+1}, E \beta\right] \equiv 0,2 J(\xi) \neq 0 \text { or }\left[\iota_{m+1}, E \beta\right] \neq 0 \text {, } \\
& 2 J(\xi) \equiv 0 \quad \bmod E \beta \circ \pi_{m+n}\left(S^{n}\right) \\
& H \text {-ımage }=Z_{2} \text { if }\left[\iota_{m+1}, E \beta\right]+2 J(\xi) \equiv 0 \text { and } 2 J(\xi) \not \equiv 0 \bmod E \beta \circ \pi_{m+n}\left(S^{n}\right) \text {, } \\
& H \text {-rmage }=\{0\} \text { otheru'se. }
\end{aligned}
$$

THEOREM 3. Suppose that the order of $\beta$ is odd. Then

and

$$
H \text {-rmage }=Z_{2} \text { if }\left[\iota_{m+1}, E \beta\right]+2 J(\xi) \equiv 0 \bmod E \beta \circ \pi_{m+n}\left(S^{n}\right)
$$

$$
H \text {-rmage }=\{0\} \text { otheruise. }
$$

Our method is based on Barcus-Barratt theory [1]. Let $A=S^{m} \bigcup_{\beta} e^{n}$ be the subcomplex of $X$ and consider the fibring

$$
r_{A}:\left(X^{X}, 1_{X}\right) \longrightarrow\left(X^{A}, i\right) \quad\left(i=1_{X} \mid A\right)
$$

defined by restricting maps on $A$. Then we have an exact sequence

$$
\pi_{1}\left(X^{A}, i\right) \underset{\partial_{X, A}}{\longrightarrow} \pi_{0}\left(r_{A}^{-1}(i), 1_{X}\right) \longrightarrow \pi_{0}\left(X^{X}, 1_{X}\right) \longrightarrow \pi_{0}\left(X^{A}, i\right) .
$$

Using an identification of $\pi_{0}\left(r_{A}^{-1}(i), 1_{X}\right)$ with $\pi_{m+n}\left(X, x_{0}\right)$, the above sequence can be transformed into the exact sequence

$$
\{0\} \rightarrow G_{X, A} \longrightarrow \mathcal{E}(X) \longrightarrow \mathcal{E}(A),
$$

where $G_{X, A}$ denotes the group $i_{*} \pi_{m+n}(A) /\left\{i_{*} \pi_{m+n}(A) \cup \partial_{X, A}\left(\pi_{1}\left(X^{A}, i\right)\right)\right.$.

Since $\mathcal{E}(A)$ can be determined by Barcus-Barratt Theorem our work is to describe the group $G_{X, A}$ and the image $\mathcal{E}(X) \rightarrow \mathcal{E}(A)$. In $\S 2$ the operation $\partial_{X, A}$ is investigated and $\S 3 \partial_{X, A}$ is considered again from the view of Suspension- 
version. $\$ 4$ contains some homotopy groups, and the image $\mathcal{E}(X) \rightarrow \mathcal{E}(A)$ is discussed in $\S 5$. At last, in $\S 6$, we give some examples.

\section{$\S 2$. Barcus-Barratt Operation.}

LEMMA 2.1. $\quad i_{*}\left(\pi_{m+n}(A)\right)=\pi_{m+n}(X), \pi_{m+n-1}(A) \cong Z\{\alpha\}+G(\beta)$ and the sequence $\{0\} \longrightarrow i_{m^{*}}\left\{\pi_{m+n-1}\left(S^{m}\right)\right\} \longrightarrow G(\beta) \longrightarrow \beta_{*}^{-1}(0) \longrightarrow\{0\}$

is exact where $\beta_{*}: \pi_{m+n-2}\left(S^{n-1}\right) \rightarrow \pi_{m+n-1}\left(S^{m}\right)$ is induced by $\beta$. Especiallly we have

$$
G_{X, A}=\pi_{m+n}(X) / \partial_{X, A} \pi_{1}\left(X^{A}, i\right) .
$$

Proof. The proof follows from the homotopy exact sequence and the homotopy excision theorem.

Let $r_{S m}:\left(X^{A}, i\right) \rightarrow\left(X^{S^{m}}, \imath_{m}\right)$ be the fibring $\left(i_{m}=\imath \mid S^{m}: S^{m} \rightarrow X\right)$ and let $\Lambda_{A, X}$ be the fibre $r_{s^{m}}^{-1}\left(i_{m}\right)$, i.e.

$$
\Lambda_{A, X}=\left\{f: A \longrightarrow X|f| S^{m}=\imath_{m}\right\} .
$$

Consider the exact sequence

$$
\pi_{1}\left(\Lambda_{A, X}, i\right) \longrightarrow \pi_{1}\left(X^{A}, i\right) \longrightarrow \pi_{1}\left(X^{S^{m}}, \imath_{m}\right) \longrightarrow \pi_{0}\left(\Lambda_{\Lambda . X}, i\right)
$$

and identifications

given by

$$
\pi_{1}\left(X^{S^{m}}, i_{m}\right) \underset{d_{1}}{\longleftrightarrow} \pi_{m+1}\left(X, x_{0}\right) \text { and } \pi_{1}\left(\Lambda_{A, X}, i\right) \underset{d_{2}}{\longleftrightarrow} \pi_{n+1}\left(X, x_{0}\right)
$$

and

$$
S^{1} \times S^{m} \underset{f}{\longrightarrow} X, \quad d_{1}(f)=d\left(f, \imath_{m} \circ p r\right)
$$

$$
S^{1} \times A \underset{g}{\longrightarrow} X, \quad d_{2}(g)=d(g, \imath \circ p r),
$$

where $d$ denotes the separation elemen (see Appendix).

LEMMA 2.2. By the composition

$$
\pi_{n+1}\left(X, x_{0}\right) \underset{d_{2}}{\longleftrightarrow} \pi_{1}\left(\Lambda_{A, X}, i\right) \longrightarrow \pi_{1}\left(X^{A}, i\right) \underset{\partial_{X, A}}{\longrightarrow} \pi_{m+n}\left(X, x_{0}\right)
$$

any element $z$ is mapped to Whitehead product $\left[\mathrm{c}_{m}, z\right]$.

For the proof we need some preparations. Let $\phi$ be a map $A \rightarrow-A \vee S^{n}$ $\left(A=S^{m} \cup e^{n} \rightarrow\left(S^{m} \cup e^{n}\right) \vee S^{n}\right)$ which is obtained from shrinking the equator of $e^{n}$ to a point.

LEMMA 2.3. $\phi_{*}(\alpha)=\alpha+\left[\iota_{m}, \iota_{n}\right]\left(\in \pi_{m+n-1}\left(A \vee S^{n}\right)\right)$

Proof. From the assumption on $m, n$ we have the decomposition

$$
\pi_{m+n-1}\left(A \vee S^{n}\right)=\pi_{m+n-1}(A) \oplus \pi_{m+n-1}\left(S^{n}\right) \oplus Z\left[\iota_{m}, \iota_{n}\right]
$$


Clearly the first factor of $\phi_{*}(\alpha)$ is $\alpha$ and the second factor is zero by the existence of the projection $X \rightarrow S^{n}$. Since the third factor is determined by the cohomology ring of $X$ we may think that it is just $\left[\iota_{m}, \iota_{n}\right]$. These complete the proof.

Let us define three spaces $X_{\imath}(i=0,1,2)$ as follows :

$$
X_{0}=\left(A \vee S^{n}\right) \bigcup_{\rho *(\alpha)} e^{m+n}, \quad X_{1}=X \vee S^{n} \quad \text { and } \quad X_{2}=S^{m} \times S^{n} \cup\left(A \vee S^{n}\right) .
$$

Then three Barcus-Barratt operations are obtained from fibrings:

$$
\left(X^{X_{\imath}}, v_{\imath}\right) \longrightarrow\left(X^{A \vee S^{n}}, \imath \vee\left(x_{0}\right)\right) \quad(i=0,1,2),
$$

where $\left(x_{0}\right)$ denotes the constant map $S^{n} \rightarrow x_{0}(\in X)$ and $v_{\imath}$ is an appropriate extension of $i \vee\left(x_{0}\right)$ over $X$. We denote them by

$$
\partial_{i}=\partial_{X_{\imath}, A \vee S^{n}}: \pi_{1}\left(X^{A \vee S^{n}}, \imath \vee\left(x_{0}\right)\right) \longrightarrow \pi_{m+n}\left(X, x_{0}\right), \quad(\imath=0,1,2) .
$$

Now, applying the additive theorem of Barcus-Barratt we have

LEMMA 2.4. $\partial_{0}=\partial_{1}+\partial_{2}$

Since $\pi_{1}\left(X^{A \vee S^{n}}, \imath \vee\left(x_{0}\right)\right)$ has a decomposition

$$
\pi_{1}\left(X^{A \vee S^{n}}, i \vee\left(\pi_{0}\right)\right)=\pi_{1}\left(X^{A}, i\right) \oplus \pi_{1}\left(X^{S^{n}},\left(x_{0}\right)\right)
$$

we may regard $\pi_{1}\left(X^{S^{n}},\left(x_{0}\right)\right)$ as a subgroup of $\pi_{1}\left(X^{A \vee S^{n}}, \imath \vee\left(x_{0}\right)\right)$.

LEMMA 2.5. The restruction $\partial_{1} \mid \pi_{1}\left(X^{S^{n}},\left(x_{0}\right)\right)=0$.

Proof. It is sufficient from definitions to show that the image of the homomorphism

$$
\pi_{1}\left(X^{X_{1}}, v_{1}\right) \longrightarrow \pi_{1}\left(X^{A \vee S} n, i \vee\left(x_{0}\right)\right)
$$

contains $\pi_{1}\left(X^{S^{n}},\left(x_{0}\right)\right)$ for the map $v_{1}: X_{1}=X \vee S^{n} \rightarrow X,\left(1_{X} \vee\left(x_{0}\right)\right)$, and then this means that any map: $S^{1} \times\left(A \vee S^{n}\right) \rightarrow X$ is extendable over $S^{1} \times\left(X \vee S^{n}\right)$ if $f \mid S^{1} \times A$ $=i \circ \operatorname{proj}_{A}$ and $f \mid * \times S^{n}=\left(x_{0}\right)$. Since the map $\tilde{f}: S^{1} \times\left(X \vee S^{n}\right) \rightarrow X$ defined by

$$
\tilde{f} \mid S^{1} \times X=1_{X} \circ \operatorname{proj}_{X} \quad \text { and } \tilde{f}\left|S^{1} \times S^{n}=f\right| S^{1} \times S^{n}
$$

satisfies the conditions the proof is completed.

LEMMA 2.6. The restriction $\partial_{2} \mid \pi_{1}\left(X^{s^{n}},\left(x_{0}\right)\right)$ can be identified with the homomorphism

$$
\pi_{1}\left(X^{S^{n}},\left(x_{0}\right)\right)=\pi_{n+1}\left(X, x_{0}\right) \longrightarrow \pi_{m+n}\left(X, x_{0}\right)
$$

defined by Whitehead product $\left[\iota_{m},\right]$.

Proof. Consider the commutative diagram 


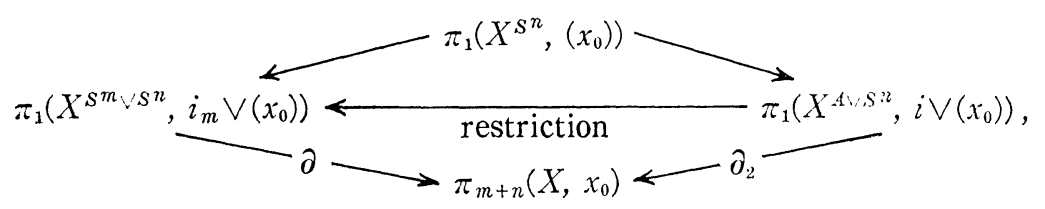

where $\partial$ on the left hand is the boundary operator derived from the fibring $X^{S^{m} \times S^{n}} \rightarrow X^{S^{m} \vee S^{n}}$. Then by Barcus-Barratt formula (p. 66 of [1]) the proof is completed.

Now, using the following diagram, the proof of lemma 2.2 is completed from lemma $2.3,2.4,2.5$ and 2.6 .

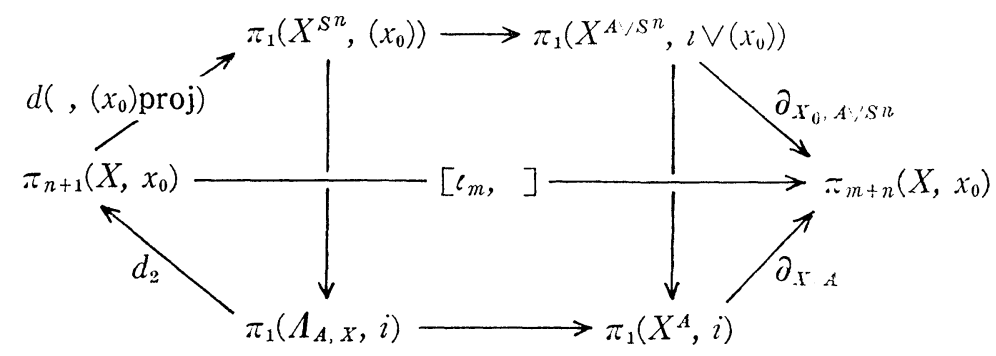

here we identify the space $A$ with $A \vee S^{n} / S^{n}$ and the map $\iota: A \rightarrow X$ with the $\operatorname{map} A \underset{\phi}{\longrightarrow} A \vee S^{n} \underset{\imath \vee\left(x_{0}\right)}{\longrightarrow}$.

\section{$\S 3$. Suspension of Barcus-Barratt Operation.}

In this section our purpose is to describe the group $E\left\{\hat{\partial}_{X, A_{A}}\left(X^{4}, i\right)\right\}$ as a subgroup of $\pi_{m+n+1}\left(E X, x_{0}\right)$ with other terms. First we consider the general case. For any spaces $Y$ and $K$, the map $\Sigma: Y^{K} \rightarrow E Y^{E K}$ which assigns each map $f: K \rightarrow Y$ to the map $E f: E K \rightarrow E Y$ induces the homomorphism

$$
\Sigma^{*}: \pi_{1}\left(Y^{K}, u\right) \longrightarrow \pi_{1}\left(E Y^{E K}, E u\right), \quad(u: K \rightarrow Y),
$$

i. e. for $f: S^{1} \times K \rightarrow Y, \Sigma *(f)(s,(t, x))=(t, f(s, x))(x \in K)$.

Since, for a map $h: L \rightarrow K$, it holds

$$
\Sigma^{*} h *(f)(s,(t, y))=(t, f(s, h(y))
$$

and

$$
(E h) * \Sigma *(f)(s,(t, y))=(\Sigma f)(s,(t, h(y))=i t, f(s, h(v)) \quad y \equiv L)
$$

we have the following commutative diagram: 


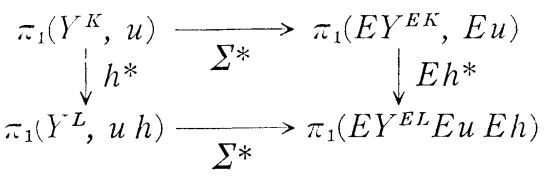

Now, applying the diagram 3.1 to our case $Y=X, K=A$ and $h=\beta$, we have

LEMmA 3.2. There exists a commutative diagram

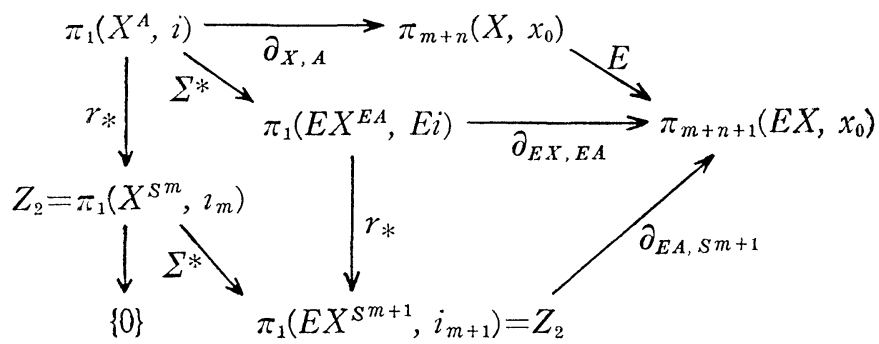
have

In the above diagram if we identify $\pi_{1}\left(E X^{S^{m+1}}, \imath_{m+1}\right)$ with $\pi_{n+2}\left(E X, x_{0}\right)$ we

LEMMA 3.3. $\hat{o}_{E-1, S m+1}$ may be considered as the composition $\circ E J(\hat{\xi})$, where $\hat{\xi}$ denotes the characteristic class of the bundle.

Proof. We note that there exists a map: $T(\xi)=S^{m+1} \bigcup_{J(\xi)} e^{m+n+1} \rightarrow E X$ of degree \pm 1 . Then the proof completed by applying the sphere theorem of [1] to the diagram

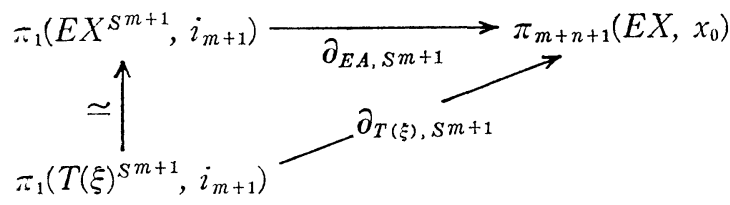

which is obtaned from using lemma 3.2.

LEMMA 3.t. $E \hat{o}_{T, 1}\left\{\pi_{1}\left(X^{A}, i\right)\right\}=\pi_{m+2}(E X) \circ E J(\xi)$.

Proof. Consider the sequence associated with the fibring $r: X^{1} \rightarrow X^{s^{m}}$

$$
\begin{aligned}
& \pi_{1}\left(\Lambda_{X \ldots}, i\right) \longrightarrow \pi_{1}\left(X^{A}, i\right) \underset{r_{*}}{\longrightarrow} \pi_{1}\left(X^{S^{m}}, \imath_{m}\right) \underset{\partial_{A, S m}}{\cong} \pi_{n}\left(X, x_{0}\right) \\
& Z_{2}=\pi_{m+1}\left(S^{n}\right) \longrightarrow \iota_{m} \circ \eta \circ E_{i} \beta
\end{aligned}
$$

Since $\hat{\partial} . \pi_{n+1}\left(S^{\eta}\right) \rightarrow \pi_{n}\left(S^{m}\right)$ is given by $\partial(\eta)=\beta \circ \eta$ and we have $\beta \circ \eta=\eta \circ E_{i} \hat{3}$, by 
the assumption $n \leqq 2 m-2 r_{*}$ is onto. Thus the proof follows from lemma 3.2 and 3.3.

\section{$\S 4$. The suspension $\pi_{k}(X) \rightarrow \pi_{k+1}(E X)$}

Now we are interested in the kernel of the suspension

$$
E_{k}: \pi_{k}(X) \longrightarrow \pi_{k+1}(E X) \quad(k=m+n, m-n-1) .
$$

Let $\nu$ be the attaching map for a cell of a $C W$-complex, then we denote by $\Sigma$ the characteristic map for the cell. By the homotopy excision we know

LEMMA 4.1. For $\imath=1,2$ there exists $a$ decomposion

$$
\begin{aligned}
\pi_{k+i}\left(E X, S^{m+1}\right) \cong & \overline{J(\xi)} \circ \pi_{k+i}\left(D^{m+n+1}, S^{m+n}\right)+\overline{E \beta} \circ \pi_{k+i}\left(D^{n-2} . S^{n}\right) \\
& +\left[\iota_{m+1}, \overline{E \beta} \circ \pi_{n+2}\left(D^{n+1}, S^{n}\right)\right]_{r},
\end{aligned}
$$

where $[,]_{r}$ denotes relative Whitehead product.

Consider the following ladder:

$$
\pi_{k+1}\left(S^{n}\right)==\pi_{k+1}\left(X, S^{m}\right) \underset{\partial}{\downarrow} \pi_{k+2}\left(E X, S^{m+1}\right) \underset{\partial}{\longrightarrow} \pi_{k+1}\left(S^{m+1}\right) \underset{\imath_{*}}{\longrightarrow} \pi_{k+1}(E X) \underset{i *}{\longrightarrow} \pi_{k+1}\left(E X, S^{m+1}\right)
$$

First we note that the homomorphism

$$
\pi_{k+i}\left(X, S^{m}\right) \longrightarrow \pi_{k+\imath+1}\left(E X, S^{m+1}\right) \quad(l=0,=1)
$$

is injective because we have a commutative diagram

$$
\begin{array}{cc}
\pi_{k+i}\left(X, S^{m}\right) \stackrel{\cong}{\longrightarrow} & \pi_{k+i}\left(S^{n}\right) \\
\downarrow_{*} E & \cong \downarrow E \\
\pi_{k+\imath+1}\left(E X, S^{m+1}\right) \stackrel{\text { Eq }}{\longrightarrow} & \pi_{k+\imath+1}\left(S^{m+1}\right) .
\end{array}
$$

Hence we have

$$
E_{k}^{-1}(0)=i_{m_{*}}\left(E^{-1} \partial \pi_{k+1}\left(E X, S^{m+1}\right)\right)
$$

On the other hand, from lemma 4.1, we have

$$
\partial \pi_{k+2}\left(E X, S^{m+1}\right)=J(\xi) \circ \pi_{k+1}\left(S^{m+1}\right) \cup E \beta \circ \pi_{k+1}\left(S^{n}\right) \cup\left[\iota_{m+1}, E j \pi_{k-m-1}\left(S^{n}\right)\right]
$$

LEMMA 4.4. For $x \in \pi_{s}\left(S^{n-1}\right)(s \leqq 2 m-2), J(\xi) \circ E^{m+1} x$ is contaned in the $E$ mage if and only if $\beta \circ x=0$.

Proof. Take Hopf invariant of the element, i.e. 


$$
H\left(J\left(\xi ; \circ E^{m-1} x\right)= \pm H J(\xi) \circ E^{m+1} x= \pm E^{m+1} \beta E^{m+1} x= \pm E^{m+1}(\beta \circ x) .\right.
$$

Then the proof follows from $s \leqq 2 m-2$.

Now, suppose that $\beta \circ x=0$. Then there exists $\sigma_{x} \in \pi_{s+1}(X)$ such that $q_{*}\left(\sigma_{x}\right)$ $=E x$. Lemma 4.4 is more exactly stated as follows :

LEMmA 4.5. There exists an element $\xi_{X} \in \pi_{s}(S O(m))$ satısfying

(1) $E J\left(\xi_{X}\right)=J(\xi) \circ E^{m+1} x$

(2) $i_{m} *\left(J\left(\xi_{x},\right)=\left[\iota_{m}, \sigma_{x}\right]\right.$

Proof. Let $\xi^{\prime}$ be the induced bundle over $S^{s+1}$ by the map Ex. Since $p_{*}\left(\xi^{\prime}\right)=p_{*}(\xi) \circ x=\xi^{\circ} \cdot x=0$ there exists an element $\xi_{X}$ of $\pi_{s}(S O(m))$ which is mapped to $\xi$ by the inclusion $S O(m) \rightarrow S O(m+1)$. Then we have

$$
E J\left(\xi_{X}\right)=-J\left(\xi^{\prime}\right)=-J(\xi \circ x)= \pm J(\xi) \circ E^{m+1} x .
$$

Next, consider the commutative diagram

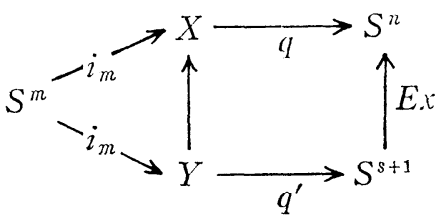

then By [2] we hare, in $\pi_{s-m}(Y)$,

$$
i_{m^{*}}\left(J\left(\xi_{X}\right)\right)+\left[\iota_{m}, \iota_{s+1}\right]=0
$$

for a cross-section $:_{0-1}$ of $q^{\prime}$. Clearly this shows (2).

Now, we know that there exists an element $u_{乏}$ of $\pi_{m+n-1}\left(S^{m}\right)$ such that

if $2 \beta=0$ then $E u_{\bar{\xi}}=\left[\iota_{m+1}, \iota_{m+1}\right] \circ E^{m+1} \beta$

if $m$ is odd ${ }^{n}$ and $2 \beta=0$ then $E w_{\xi}=J(2 \xi) \pm\left[\iota_{m+1}, c_{m+1}\right] \cdot E_{i} \beta$.

Then from $4.2:,(4.3)$, and lemma 4.5 we obtain

LEMMA 4.6. $E_{m-n}^{-1}(0)=\left[\iota_{m}, \pi_{n+1}(X)\right] \cup\left\{l_{m *}\left(w_{\xi} \circ \eta\right)\right\}$

$$
E_{m-n-1}^{-1}(0)=\left[\ell_{m}, \pi_{n}(X)\right] \cup\left\{i_{m^{*}}\left(u_{\xi}\right)\right\} \text {. }
$$

Lemma 4.7. Suppose that $\left[c_{m+1}, E \beta\right] \circ \eta \equiv 0 \bmod E \beta \circ \pi_{m+n+1}\left(S^{n}\right)$. Then we have

$$
\hat{o}_{\mathrm{I} .1 .} \pi_{1}\left(X^{-1}, i\right)=\left\{\left[\ell_{m}, \pi_{n+1}(X)\right]\right\} \cup\left\{\pi_{m+1}\left(S^{m}\right) \circ J(\xi)\right\} .
$$

Proof. By lemma 3.4 there exists an element $\boldsymbol{\gamma}_{;}$of $\pi_{1}\left(X^{A}, i\right)$ satisfying

(1) $E \partial_{x, A}\left(\gamma_{\xi}\right)=i_{m+2 *}(\eta) \circ E J(\xi)$

(2) $\gamma_{\xi}$ is mapped to the generator of $\pi_{1}\left(X^{S^{m}}, i_{m}\right)=Z_{2}$ by $r_{*}$. 
Since $\pi_{1}\left(X^{A}, i\right)$ is the sum of $\left\{\gamma_{\xi}\right\}$ and the image $\pi_{1}\left(\Lambda_{X, A}, i\right) \rightarrow \pi_{1}\left(X^{A}, i\right)$ the proof is completed by lemma 2.6 and 4.6 .

\section{§. Proof of theorems.}

Recall the sequence in $\S 1$

$$
0 \longrightarrow G_{X, A} \longrightarrow \mathcal{E}(X) \longrightarrow \mathcal{e}(A),
$$

and imbed this one in a diagram as follows:

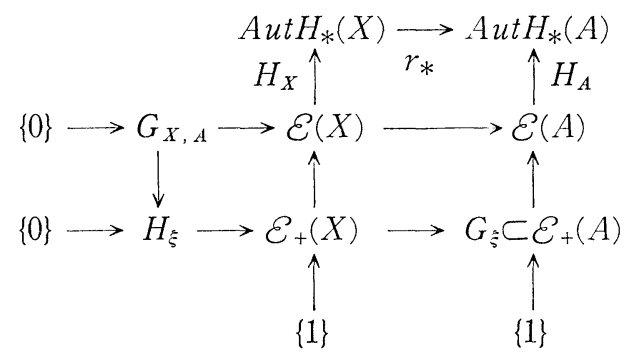

Then, if $\left[\iota_{m+1}, E \beta \circ \eta\right] \in E \beta \circ \pi_{m+n+1}\left(S^{n}\right)$, we have from lemma 2.1 and 4.7

LEMMA 5.1. $H_{\xi}=\pi_{m+n}(X) /\left\{\left[\iota_{m}, \pi_{n+1}(X)\right]\right\} \cup\left\{\pi_{m+1}(X) \circ J(\xi)\right\}$

Next, consider the exact sequence

which is defined by

$$
\pi_{n}\left(S^{m}\right) \longrightarrow{ }_{t} \mathcal{C}(A) \longrightarrow Z_{2} \times Z_{2}
$$

$$
t(f): A \longrightarrow \underset{\phi}{\longrightarrow} A \vee S^{n} \underset{1 \vee f}{\longrightarrow} A \vee S^{n} \underset{1 \vee \imath_{m}}{\longrightarrow} A \quad\left(f \in \pi_{n}\left(S^{m}\right)\right)
$$

and $d(h)=\left(\right.$ degree on $e^{m}$ of $h$, degree on $e^{n}$ of $h$ ).

Clearly $d$ is equivalent to the representation $H$ and moreover the kernel of $t$ is determined by the sphere theorem of [1] as follows :

$$
t^{-1}\left(1_{X}\right)=\{\eta \circ E \beta\}=\{\beta \circ \eta\} \text {. }
$$

Since the definition of $t$ and lemma 2.3 imply

$$
t(f)_{*}(\alpha)=\alpha+\left[\iota_{m}, f\right] \quad\left(X=A \bigcup_{\alpha} e^{m+n}\right)
$$

the element $t(f)$ is contained in the image $\varepsilon(X) \rightarrow \varepsilon(A)$ if and only if $\left[\iota_{m}, f\right] \in$ $\partial \pi_{m+n}\left(S^{n}\right)=\beta \circ \pi_{m+n-1}\left(S^{n-1}\right)$.

Thus, noting $r H_{X}=H_{A} r$, we have

LEMMA 5.2. $G_{\xi}=P_{n}^{m}(\beta) /\{\beta \circ \eta\}$ if $\left[\ell_{m+1}, E \beta \circ \eta\right] \in E \beta \circ \pi_{m+n+1}\left(S^{n}\right)$

Now we proced to study of the representation $H_{X}$. First we note 
LEMmA 5.3. The kernel $(q \mid A)_{*}: \pi_{m+n-1}(A) \rightarrow \pi_{m+n-1}\left(S^{n}\right)$ is generated by $\alpha$ and the $\imath_{m *}$-image $\left(i_{m}: S^{m} \rightarrow A\right)$.

Proof. This is easily obtained from the diagram $(k=m+n-1)$

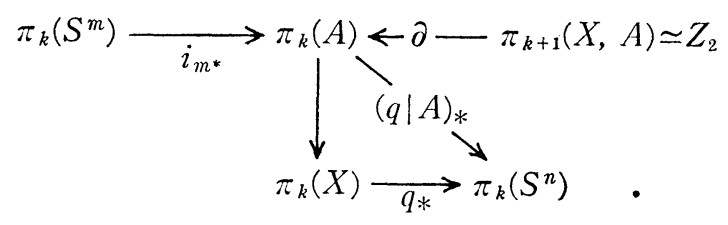

Let $f$ be a map : $A \rightarrow A$ satisfying

$$
f_{*}\left(e^{m}\right)=a e^{m} \quad \text { and } \quad f_{*}\left(e^{n}\right)=b e^{n}
$$

which we call a map of type $(a, b)$ and denote by $f_{a}^{b}$. Then the following lemma is easy.

LEMmA 5.4. There exists a map of type $(a, b)$ if and only if $(b-a) \beta=0$.

Let $g_{a}^{b}$ be another map. Clearly there exists a map $g: S^{n} \rightarrow S^{m}$ by which $g_{a}^{b}$ is represented as the composition of maps

$$
g_{a}^{b}=\left(f_{a}^{b} \vee g\right) \circ \phi: A \longrightarrow{ }_{\phi} \rightarrow A \vee S^{n} \underset{f_{a}^{b} \vee g}{\longrightarrow} A \vee S^{m} \longrightarrow A
$$

Now we are interested in the element $f_{a^{*}}^{b}(\alpha)$. Then lemma 2.3 gives

$$
g_{a *}^{b}(\alpha)=f_{a *}^{b}(\alpha)+a\left[\iota_{m}, g\right] .
$$

On the other hand, since we have

$$
(q \mid A)_{*} f_{a *}^{b}(\alpha)=\left(b \iota_{n}\right)_{*}(q \mid A)_{*}(\alpha)=0
$$

lemma 5.3 gives, for some $\sigma_{a}^{b} \in \pi_{m+n-1}\left(S^{m}\right)$,

$$
f_{a^{*}}^{b}(\alpha)=a b \alpha+\imath_{m^{*}}\left(\sigma_{a}^{b}\right) .
$$

Thus we have from these lemmas

Lemma. 5.5. There exists a map $f: X \rightarrow X$ whose restriction $f \mid A$ is of type $(a, b)$ if and only if there exists a map $f_{a}^{b}$ such that

$$
f_{a^{*}}^{b}(\alpha)=a b \alpha+\imath_{m^{*}}\left(\sigma_{a}^{b}\right), \quad \sigma_{a}^{b} \in a\left[\iota_{m}, \pi_{n}\left(S^{m}\right)\right] \cup \beta \circ \pi_{m+n-1}\left(S^{n-1}\right) .
$$

Especially if $a= \pm 1$ the condition is equivalent to $E \phi_{a}^{b} \in E \beta \circ \pi_{m+n}\left(S^{n}\right)$.

Next, for the reason of our dimensional assumption, the space $A$ is desuspendable, so there exists a co- $H$-map $\nu: A \rightarrow A \vee A$ and the addition of two maps is defined as usal. Then we want to get some formula on $\left(f_{a}^{b}+f_{c}^{d}\right)_{*}(\alpha)$. For the 
purpose we must investigate the group $\pi_{k}(A \vee A)$ for $k=m+n-1$. First, by the well-known decomposition of this group it holds

$$
\nu_{*}(\alpha)=\alpha+\alpha+\chi \quad\left(\chi \in \partial \pi_{k+1}(A \times A, A \vee A)\right) .
$$

Next- since the order of $\beta$ 1s finite there exists a map $\tau: S^{n} \rightarrow A$ of degree $o(\beta)$ and we have the element $\left[\iota_{m}^{1}, \tau^{2}\right]$ of $\pi_{k}(A \vee A)$ where each upper index denotes the order of $A$ imbedded in $A \vee A$ and $o(\beta)$ is the order of the element. Let $Q: A=S^{m} \cup e^{n} \rightarrow S^{n}=A / S^{m}$ be the collapsing map, then for maps $Q \vee 1_{A}: A \vee A$ $\rightarrow S^{n} \vee A$ and $1_{A} \vee Q: A \vee A \rightarrow A \vee S^{n}$ we have

LEMMA 5.6. $\left(1_{A} \vee Q\right)_{*}(\mathcal{X})=\left[\iota_{m}^{1}, \iota_{n}\right], \quad\left(Q \vee 1_{A}\right)_{*}(\mathcal{X})=(-1)^{m n}\left[\iota_{n}, \iota_{m}^{2}\right]$,

$$
\left(1_{A} \vee Q\right)_{*}\left(\left[\iota_{m}^{1}, \tau^{2}\right]\right)=0(\beta)\left[\iota_{m}^{1}, \iota_{n}\right] \text { and }\left(Q \vee 1_{A}\right)_{*}\left(\left[\iota^{1}, \tau_{m}^{2}\right]\right)=0(\beta)\left[\iota_{n}, \iota_{m}^{2}\right] \text {. }
$$

Proof. The third and fourth are clear and the others follows from the diagram

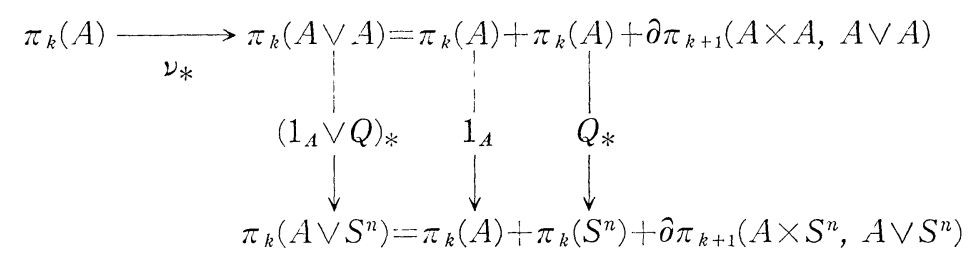

LEMMA 5.7. There exists an isomorphism

$$
\pi_{k}(A \vee A)=\pi_{k}(A)+\pi_{k}(A)+Z\{\chi\}+Z\left[\tau^{1}, \iota \frac{2}{m}\right]+\left[\iota_{m}^{1}, \iota_{m}^{2}\right] \circ \pi_{k}\left(S^{2 m-1}\right)
$$

Proof. Noting the assumption $m+1<n<2 m-1$, consider the following diagram which is naturally obtained:

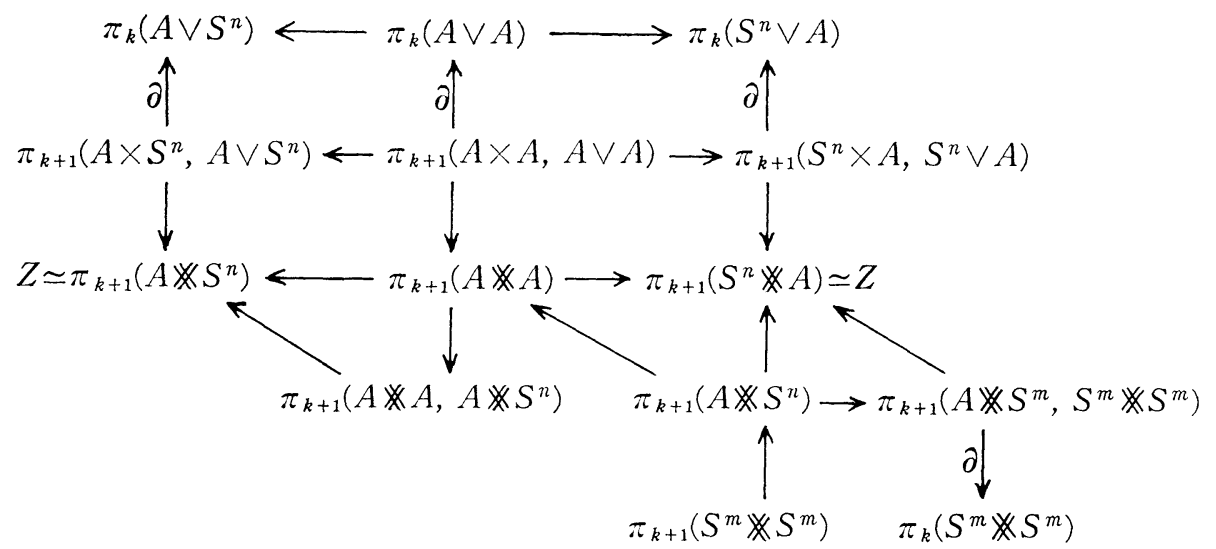

, where denotes the reduced join operator. 
Then the proof follows from lemma 5.6.

Now, consider the map $f_{a}^{b} \vee f_{c}^{d}: A \vee A \rightarrow A \vee A$, then we prove

LEMMA 5.8. $\left(f_{a}^{b} \vee f_{c}^{d}\right)_{*}\left(\left[\tau^{1}, \iota_{m}^{2}\right]\right) \equiv b c\left[\tau^{1}, \iota_{m}^{2}\right] \quad \bmod \left[\iota_{m}^{1} \circ \pi_{n}\left(S^{m}\right), \iota_{m}^{2}\right]$ and

$$
\left(f_{a}^{b} \vee f_{c}^{d}\right)_{*}(\chi) \equiv a d\{\chi\}+\left\{(-1)^{m n}(b c-a d) / 0(\beta)\right\}\left[\tau^{1}, \iota_{m}^{2}\right] \quad \bmod \left[\iota_{m}^{1}, \iota_{m}^{2}\right] \circ \pi_{k}\left(S^{2 m-1}\right)
$$

Remark. $b c-a d$ is divisible by $o(\beta)$ because we have $b-c \equiv 0 \equiv d-a \bmod o(\beta)$ (lemma 5.4).

Proof. By lemma 5.7 we can put

$$
\left(f_{a}^{b} \vee f_{c}^{d}\right)_{*}(\chi) \equiv r\{\chi\}+s\left[\tau^{1}, \iota_{m}^{2}\right] \quad \bmod \left[\iota_{m}^{1}, \iota_{m}^{2}\right] \circ \pi_{k}\left(S^{2 m-1}\right)
$$

for some integers $r$ and $s$. Then from lemma 5.6 it follows that $r=a d$ and $o(\beta) s=(-1)^{m n}(b c-\gamma)=(-1)^{m n}(b c-a d)$. Hence the proof is completed.

Let $\mu$ be the folding map $A \vee A \rightarrow A$. We note that there exists an element $\lambda \equiv \pi_{n-1}(S O(m))$ such that

$$
o(\beta) \alpha=\imath_{m}(J(\lambda))+\left[\iota_{m}, \tau\right]
$$

where $i_{*}(\lambda)=o(\beta) \xi$ for $\imath: S O(m) \rightarrow S O(m+1)$.

LEMMA 5.9. $\mu_{*}(\chi)=2 \alpha+\imath_{m^{*}}\left(\sigma_{2}^{2}\right)$ and $\mu_{*}\left(\left[\tau^{1}, \iota_{m}^{2}\right]\right)=(-1)^{m n}\left\{o(\beta) \alpha-\imath_{m^{*}}(J(\lambda))\right\}$

Proof. By definition $\nu_{*}(\alpha)=\alpha+\alpha+\chi$, then we have

$$
\mu_{*} \nu_{*}(\alpha)=\left(2 \cdot 1_{A}\right)_{*}(\alpha) \text { i. e. } 4 \alpha+i_{m^{*}}\left(\sigma_{2}^{2}\right)=2 \alpha+\mu_{*}(\chi) .
$$

Since $\mu_{*}\left(\left[\tau^{1}, \iota_{m}^{2}\right]\right)=\left[\tau, \iota_{m}\right]$ the second follows from the above note.

LEMMA 5.10. $\quad \sigma_{a+c}^{b+d} \equiv \sigma_{a}^{b}+\sigma_{c}^{d}+a d\left(\sigma_{2}^{2}\right)+\{(a d-b c) / o(\beta)\} J(\lambda)$

$$
\bmod \left[\iota_{m}, \pi_{n}\left(S^{m}\right)\right] \cup\left\{\beta \circ \pi_{m+n-1}\left(S^{n-1}\right)\right\}
$$

Proof. Apply above lemmas to the identity

$$
\left(f_{a+c}^{b+d}\right)_{*}(\alpha)=\left(f_{a}^{b}+f_{c}^{d}\right)_{*}(\alpha)=\mu_{*}\left(f_{a}^{b} \vee f_{c}^{d}\right)_{* \nu_{*}}(\alpha) .
$$

Then the proof easily follows.

Now, let $\hat{\sigma}_{a}^{b}$ be the suspension of $\sigma_{a}^{b}$, then lemma 5.10 gives rise

$$
\hat{\sigma}_{a+c}^{b+d} \equiv \hat{\sigma}_{a}^{b}+\hat{\sigma}_{c}^{d}+a d\left(\hat{\sigma}_{2}^{2}\right)-(a b-c d) J(\xi) \bmod E \beta \circ \pi_{m+n}\left(S^{n}\right) .
$$

LEMMA 5.11. $\hat{\sigma}_{a}^{b}=\{a(a--1) / 2\}\left(\hat{\sigma}_{-1}^{-1}\right)+a(b-a) J(\xi) \quad \bmod E \beta \circ \pi_{m+n}\left(S^{n}\right)$

Proof. By lemma $5.4 b=a+k o(\beta)$ for some integer $k$. Hence we have

$$
\widehat{\sigma}_{a}^{b}=\hat{\sigma}_{0+a}^{k o(\beta)+a}=\hat{\sigma}_{0}^{k o(\beta)}+\widehat{\sigma}_{a}^{a}+k o(\beta) J(\xi)=\hat{\sigma}_{a}^{a}+a(b-a) J(\xi) \bmod E \beta \cdot \pi_{m+n}\left(S^{n}\right)
$$


On the other hand, lemma 5.10 implies $\hat{\sigma}_{a+1}^{a+1} \equiv \hat{\sigma}_{a}^{a}+a\left(\widehat{\sigma}_{2}^{2}\right)$, i. e. we have

$$
\hat{\sigma}_{a}^{a}=\{a(a-1) / 2\}\left(\hat{\sigma}_{-1}^{-1}\right) .
$$

Thus the proof is completed.

Since lemma 5.11 shows that it is important for our purpose to determine $\hat{\sigma}_{-1}^{-1}$, so here we recall the definition of $\sigma_{-1}^{-1}$, which is given by

$$
\left(-1_{A}\right)_{*}(\alpha)=\alpha+i_{m *}\left(\sigma_{-1}^{-1}\right) \text {. }
$$

Then, applying the suspension operator, we have

$$
\left(-1_{E A}\right)_{*}(E \alpha)=E \alpha+i_{m+1^{*}}\left(\hat{\sigma}_{-1}^{-1}\right) \text {, i. e. } i_{m+1^{*}}\left(\hat{\sigma}_{-1}^{-1}\right)=-2 E \alpha .
$$

On the other hand, since we may regard the mapping cone of the projection $q: X \rightarrow S^{n}$ as the Thom space of the vector bundle characterized by $\xi$ we can put

$$
E \alpha=\imath_{m+1 *}\left(\lambda_{\xi} J(\xi)\right), \quad \lambda_{\xi}=1 \text { or }-1 .
$$

Hence, using $\imath_{m+1 *}^{-1}(0)=\left\{\left[\iota_{m+1}, E \beta\right]\right\} \cup E \beta \circ \pi_{m+n}\left(S^{n}\right)$, we know that

$$
\hat{\sigma}_{-1}^{-1} \equiv-2 \lambda_{\xi} J(\xi)+c_{\hat{\xi}}\left[\iota_{m+1}, E \beta\right] \bmod E \beta \circ \pi_{m+n}\left(S^{n}\right)
$$

for some integer $c_{\hat{\xi}}$. For example, if $\xi$ has a cross-section then we may take $\lambda_{\xi}=-1$ ([2]), but, in general, it is not easy to determine $\lambda_{\xi}$.

LEMMA 5.13. $o(\beta)\left(1+\lambda_{\xi}\right) J(\xi)=0$

Proof. Consider the following diagrams $(a=o(\beta))$ :

and
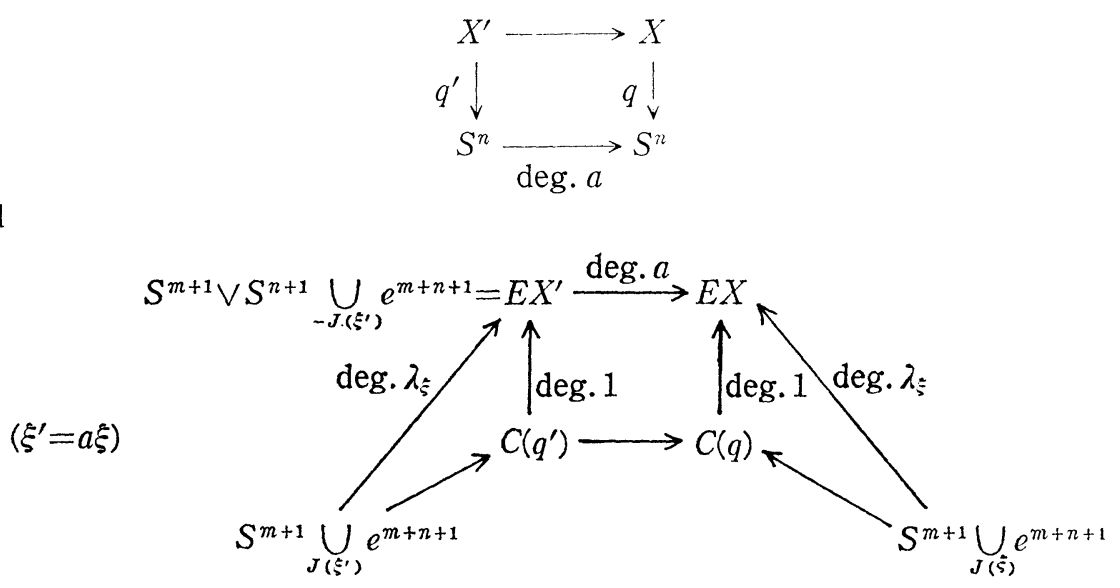

Then we can obtain

$$
a \equiv a \lambda_{\xi} \lambda_{\xi^{\prime}} \quad \bmod o(J(\xi)) \text { and }-\lambda_{\xi^{\prime}} J\left(\xi^{\prime}\right)=J\left(\dot{\xi}^{\prime}\right), \text { i. e. } \quad-\lambda_{\xi^{\prime}} a J(\xi)=a J\left(\xi_{\xi}\right) \text {. }
$$

Clearly these give the proof. Now we prove 
LEMMA 5.14 In (5.12) we can take

(1) $\lambda_{\bar{\xi}}=-1$ and $c_{\xi}=1$ if $2 \beta=0$

(2) $c_{\xi}=-\lambda_{\xi}$ or $-\lambda_{\xi}+o(\beta) / 2$ otherwise

Proof. (1) the case : $2 \beta=0$.

By lemma 5.13 and 5.11 we have

$2 J(\xi)=-2 \lambda_{\xi} J(\xi)$ and $\hat{\sigma}_{2}^{0} \equiv \hat{\sigma}_{-1}^{-1}-4 J(\xi)$. On the other hand, $f_{2^{*}}^{0}(\alpha)=\sigma_{:}^{0}$

implies that

$$
\hat{\sigma}_{2}^{0}=\left(E f_{2}^{0}\right)_{*}(E \alpha)=2 \lambda_{\xi} J(\xi)+\left[\iota_{m+1}, \iota_{m+1}\right] H J(\xi)=2 \lambda_{\xi} J(\xi)+\left[\iota_{m+1}, E \beta\right]
$$

Hence we obtain

$$
\hat{\sigma}_{-1}^{-1} \equiv 4 J(\xi)+2 \lambda_{\xi} J(\xi)+\left[\iota_{m+1}, E \beta\right] \equiv 2 J(\xi)+\left[\iota_{m+1}, E \beta\right] .
$$

(2) the other case. Note that this occurs only in the case of $m=$ odd.

Take Hopf-invariant on the both side of (5.12), then we have, from the formula $H(J(\xi))=-E^{m+1}(\xi)$ and $H\left(\left[\iota_{m+1}, E \beta\right]\right)=2 E^{m+1} \beta$,

$$
2 \lambda_{\xi} E^{m+1}+2 c_{\xi} E^{m+1} \beta=0 \text {. }
$$

Then, in our dimensional restriction, this means $2\left(\lambda_{\xi}+c_{\xi}\right)=0$ and then the proof is completed.

Now the proof of theorem 2 and 3 are completed by the following lemma which is a conseqeuence of lemma 5.11 and 5.14 .

LEMMA 5.15. If $2 \beta=0$ we have

$$
\begin{aligned}
& \hat{\sigma}_{-1}^{-1} \equiv 2 J(\xi)+\left[\iota_{m+1}, E \beta\right] \\
& \hat{\sigma}_{1}^{-1} \equiv-2 J(\xi) \\
& \hat{\sigma}_{-1}^{1} \equiv\left[\iota_{m+1}, E \beta\right] \bmod E \beta \circ \pi_{m+n}\left(S^{n}\right)
\end{aligned}
$$

and if the order of $\beta$ is odd

$$
-\lambda_{\xi} \hat{\sigma}_{-1}^{-1} \equiv 2 J(\xi)+\left[\iota_{m+1}, E \beta\right] .
$$

Remark. Since the second case of lemma 5.15 can be shown to be true in the case $o(\beta)=2 \cdot$ odd Theorem 3 also holds in this case.

\section{\$. Some Examples}

(1) The case of having a cross section.

$$
\begin{aligned}
& H_{\xi}=\pi_{m+n}\left(S^{m}\right) /\left\{\eta \circ J(\xi) \cup\left[\iota_{m}, \pi_{n+1}\left(S^{m}\right)\right]\right\}+\pi_{m+n}\left(S^{n}\right) . \\
& G_{\xi}=\left\{x \mid x \in \pi_{n}\left(S^{m}\right),\left[\iota_{m}, x\right]=0\right\}
\end{aligned}
$$


and

$$
\mathcal{E}(X) \longrightarrow Z_{2} \quad \text { is onto if } 2 J(\xi) \neq 0
$$

$$
\mathcal{E}(X) \longrightarrow Z_{2} \times Z_{2} \quad \text { is onto if } 2 J(\xi)=0 \text {. }
$$

(II) Complex Stiefel manifolds $W_{n, 2}(n \geqq 5)$.

Let $\xi_{n}$ be the standard sphere bundle

$$
S^{2 n-3} \longrightarrow W_{n, 2} \longrightarrow S^{2 n-1}, \quad \beta_{n}=n \eta \text {. }
$$

Since $\left[\iota_{2 n-2}, \eta \circ \eta\right]=\eta \circ\left[\iota_{2 n-1}, \iota_{2 n-1}\right]$ the assumption is satisfied. If $n$ is even the case reduces to (I) and for odd $n$ we have

if $n \equiv 1 \bmod 4$, then $H_{\tilde{\xi}_{n}}=\pi_{1 n-1}\left(W_{n, 2}\right), \quad G_{\tilde{⿱}_{n}}=\{0\}$ and $\mathcal{E}\left(W_{n, 2}\right) \longrightarrow Z_{2.2}$ is onto. and

if $n \equiv 3 \bmod 4$, then $H_{\bar{\xi}_{n}}=\pi_{4 n-4}\left(W_{n, 2}\right) / \imath *\left\{\left[\iota_{2 n-3}, \pi_{2 n}\left(S^{2 n-3}\right)\right]\right\}$,

$$
G_{\xi_{n}}=\{0\}, \quad \varepsilon\left(W_{n, 2}\right) \rightarrow Z_{2} \text { is onto. }
$$

(III) Quaternion Stiefel manifolds $X_{n, \text { }}$

Let $\tau_{n}$ be the standard bundle

$$
S^{4 n-5} \longrightarrow X_{n, 2} \longrightarrow S^{1 n-1}, \quad \tau_{n}=n \nu .
$$

Since $\left[\iota_{4 n-4}, \nu \circ \eta\right]=0$ the assumption is satisfied. Then if $n \geqq 3$ we have

$$
H_{\tau_{n}}=\pi_{8 n-6}\left(X_{n, 2}\right) /\left[\iota_{4 n-5}, \pi_{+n}\left(X_{n, 2}\right)\right] \cup_{\imath_{*}}\left\{\eta \circ J\left(\tau_{n}\right)\right\} \text {, and } G_{\tau_{n}}=\{0\} \text {. }
$$

The image $\varepsilon\left(X_{n, 2}\right) \rightarrow Z_{2} \times Z_{2}$ is more complicated, so we omit it.

\section{APPENDIX: Separation elements}

$$
\begin{array}{ll}
K \cup e^{n-f} \longrightarrow X, & f|K=g| K \Rightarrow d(f, g) \in \pi_{n}(X) . \\
K \cup e_{\imath}^{n}=K \cup e^{n}, & \hat{K}=e_{1}^{n} \cup K \cup e_{. .}^{n}, \\
k: \hat{K} \longrightarrow K \cup e^{n}, & k \mid K \cup e_{\imath}^{m}=\text { identity. }
\end{array}
$$

1. The sequence $: 0 \rightarrow \pi_{n}(\hat{K})-\rightarrow \pi_{n}(\hat{K}, K) \times \pi_{n}\left(K \cup e^{n}\right)$ is exact.

For,

where $\imath: K \cup e^{n} \rightarrow e_{1}^{n} \cup K \subset \hat{K}$.

$$
\begin{aligned}
\pi_{n}(K) \longrightarrow & \pi_{n}(\hat{K}) \longrightarrow \pi(K, \hat{K}) \\
& k_{*} \downarrow \uparrow \imath_{*} \\
& \pi_{n}\left(K \cup e^{n}\right)
\end{aligned}
$$

$$
\begin{aligned}
& \xi: S^{n}=E_{\perp}^{n} \cup E^{n} \longrightarrow D^{n}-\chi_{1} \longrightarrow K . \\
& \square k_{*}(\xi)=0, \quad \chi_{1}-\chi_{2} \in \pi_{n}(K, K) .
\end{aligned}
$$


Then $d(f, g)=h_{*}(\xi)$, where $h: \hat{K} \rightarrow X, h\left|e_{1}^{n} \cup K=f, h\right| K \cup e_{2}^{n}=g$.

2. $K \cup e^{n} \underset{H}{\longrightarrow} e_{\lambda_{1}}^{n} \bigcup_{\lambda_{1}} L e_{2}^{n} \succ F>X$,

$$
H(K) \subset L, H\left(e^{n}\right)=e_{1}^{n}+e_{2}^{n}, \quad F|L=G| L .
$$

Then $d(F H, G H)=d\left(f_{1}, g_{1}\right)+d\left(f_{2}, g_{2}\right)$, where $f_{\imath}=F \mid e_{\imath}^{n} \cup L$ and $g_{2}=G \mid e_{\imath}^{n} \cup L$.

Proof.

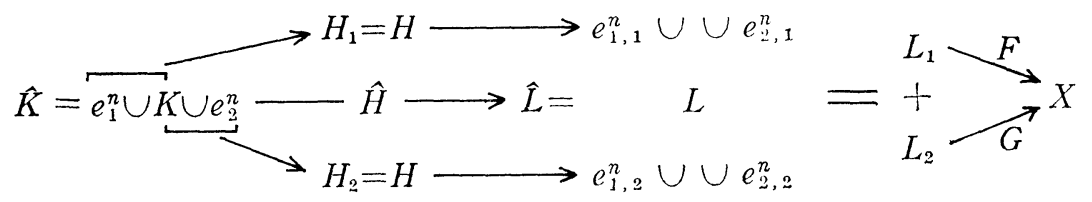

And consider the diagram :

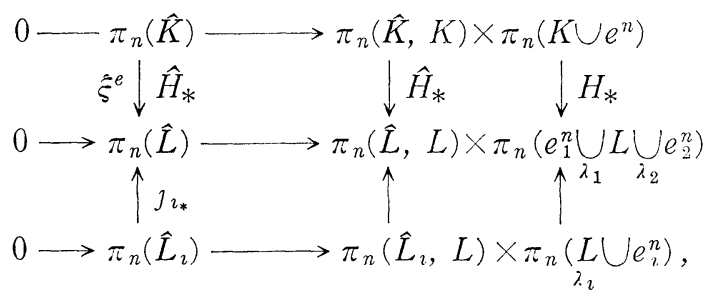

where

Then

$$
\hat{L}_{\imath}=e_{\imath, 1}^{n} \bigcup_{\imath, 1} L \bigcup_{\lambda_{\imath, 2}} e_{\imath, 2}^{n} \longrightarrow \jmath_{\imath} \longrightarrow \hat{L}
$$

$$
\begin{aligned}
& H_{*}(\xi) \longrightarrow\left(\lambda_{1,1}+\lambda_{2,1}-\lambda_{1,2}-\lambda_{2,2}\right) \times 0 \\
& \jmath_{1 *}\left(\xi_{1}\right)+\jmath_{2 *}\left(\xi_{2}\right) \longrightarrow\left(\lambda_{1,1}-\lambda_{1,2}+\lambda_{2,1}-\lambda_{2,2}\right) \times 0
\end{aligned}
$$

Hence $\hat{H}_{*}(\xi)=\jmath_{1_{*}}\left(\xi_{1}\right)+\jmath_{2 *}\left(\xi_{2}\right)$ (from the injectivity). And then we have

$$
\begin{aligned}
d(F H, F G) & =(F \cup G)_{*} H_{*}(\xi) \\
& =(F \cup G)_{*}\left(\jmath_{1_{*}}\left(\xi_{1}\right)\right)+(F \cup G)_{*}\left(\jmath_{2 *}\left(\xi_{2}\right)\right) \\
& =d\left(f_{1}, g_{1}\right)+d\left(f_{2}, g_{2}\right) .
\end{aligned}
$$

\section{REFERENCES}

[1] Barcus. W.D. ANd M. G. Barratt, On the homotopy classification of the extension of a fixed map, Trans. Amer. Math. Soc., 88 (1958), 57-74.

[2] I.M. James AND J.H.C. Whitehead, The homotopy of sphere bundles over spheres (I), Proc. Lond. Math. Soc., 4 (1954), 196-218. 
[3] J.W. RutTer, The group of self-homotopy equivalences of principal three sphere bundles over the seven sphere, Math. Proc. Camb. Phil. Soc., 84 (1978), 303-311.

[4] S. SASAO, On self-homotopy equivalences of $S^{3}$-principal bundles over $S^{n}$. (to appear)

[5] Y. NOMURA, Self-homotopy equivalences of Stiefel manifolds $W_{n, 2}$ and $V_{n, 2}$, Osaka J. Math., 20 (1983), 79-93.

[6] M. Mimura and N. Sawashita, On the group of self-homotopy equivalences of principal $S^{3}$-bundles over spheres. (to appear in Hiroshima Math. J.)

DePt. OF MATH.

TOKyo Inst. Tecil.

Oh-okayama Meguro-ku

TOKYO JAPAN 